\title{
Capturing a Total Solar Eclipse
}

\author{
Joo Beng Koh ${ }^{1, *}$ \\ ${ }^{1}$ Institute of Physics, Singapore
}

\begin{abstract}
A Total Solar Eclipse is one of the astronomical world's most awe-inspiring phenomena. It is a relatively rare astronomical event that lasts at most a few minutes and can be viewed only at very specific times and from very specific locations on the globe. Capturing and documenting the event so that it can be shared with others is exceptionally challenging because of its rarity and brevity. This article addresses some of the challenges and possible solutions with the use of various forms of technology as well as program management skills.
\end{abstract}

\section{Total Solar Eclipse}

A total solar eclipse is a relatively rare event and only a very small proportion of people actually experience one. More commonly experienced are lunar eclipses and partial solar eclipses, and yet total solar eclipses are not as rare as one may imagine it to be. It happens on average every 1 to 2 years ${ }^{\dagger}$, but yet it is so rare because it is only visible from a very narrow path on the Earth and lasts at most 2 to 3 hours. Totality itself, which is the critical period during the eclipse when the Sun is completely obscured by the Moon, is even more fleeting, lasting 2 to 3 minutes on average, and at most about 6 and a half minutes.

\subsection{Geometry of Eclipses}

Eclipse geometries are determined by the positions of the Earth, the Sun, and the Moon in space. Solar eclipses occur when the Moon is between the Earth and Sun. As the Moon revolves around the Earth about 13 times a year, it may be thought that there would be 13 solar eclipses each year. However, due to the $5^{\circ}$ tilt of the Moon-Earth plane relative to the Sun-Earth plane (see Fig. 1), there are only 2 opportunities for a Solar eclipse to occur each year, and can happen only when the Moon is at the intersection of the Moon-Earth plane with the Sun-Earth plane.

With the Moon between the Sun and the Earth, the distance of the Moon from the Earth now comes into play. As the Moon's orbit is slightly elliptical, its distance from the Earth varies as it orbits. This variation in distance results in annular, hybrid, or total eclipses. Total eclipses occur when the Moon is so close to the Earth that the Sun is able to cast an umbral shadow on the Earth's surface; Annular eclipses occur when the Moon is so far away that the Sun only casts an antumbral shadow; and a hybrid eclipse occurs when the Sun casts an

\footnotetext{
*Email : joobeng@astrobargains.com

$\dagger$ 1991-2000: 7 total eclipses, 2001-2010: 7 total eclipses, 2011-2020: 6 total eclipses, Average: 20 total eclipses in 30 years, or 1.5 years per eclipse.
} 


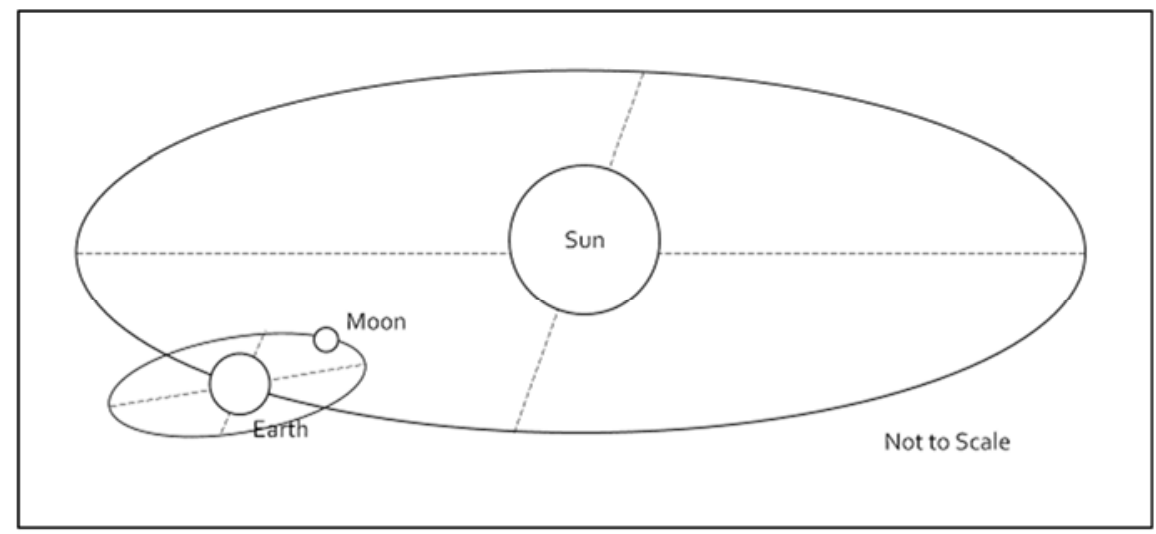

Fig. 1. The Earth-Moon orbit is tilted relative to the Sun-Earth orbit

umbral shadow along a portion of the eclipse path and antumbral shadow for the rest of its path (Fig. 2).

\subsubsection{Width of a total eclipse path}

The width of an eclipse path is determined by many of the factors discussed so far as well as where the umbral shadow falls upon the Earth. The path will be wider when it falls closer to the edges of the globe (see Fig. 3). The widest that a path on the equator can be (so that we can ignore the curvature of the Earth) is when the Moon is at perigee, the Sun at perihelion, and works out to be just about $100 \mathrm{~km}$. Theoretically, if this path is projected at the edges of the globe, it could span about $1000 \mathrm{~km}$, so that would be roughly the maximum width.

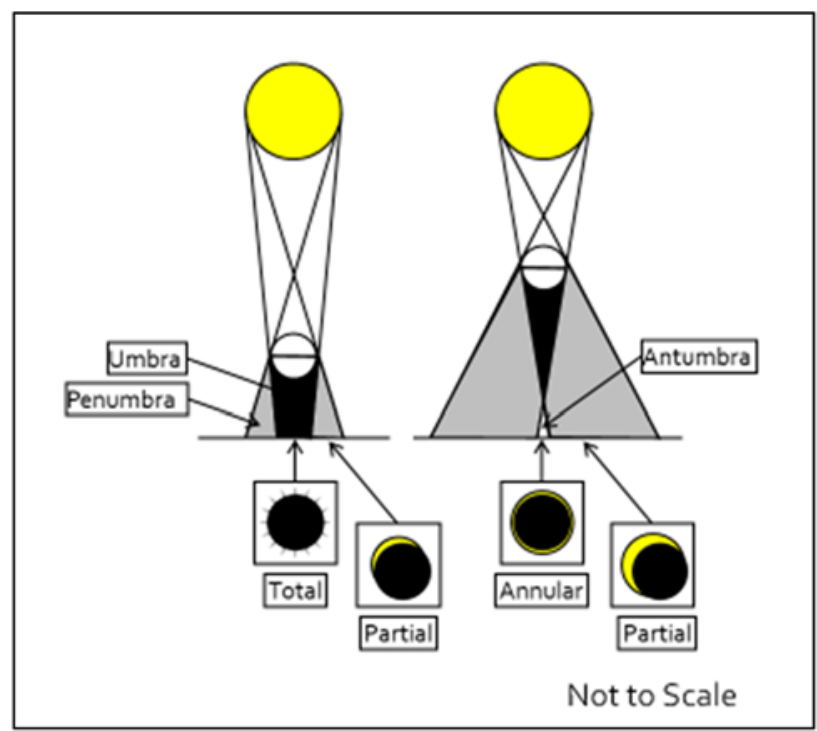

Fig. 2. Types of Solar Eclipses

\footnotetext{
Assumptions: Distance of Moon at perigee $=356400 \mathrm{~km}$, Diameter of Moon $=3474 \mathrm{~km}$, Distance of Sun at perihelion $=1.471 \mathrm{e} 8 \mathrm{~km}$, Diameter of $\operatorname{Sun}=1.391 \mathrm{e} 6 \mathrm{~km}$.
} 


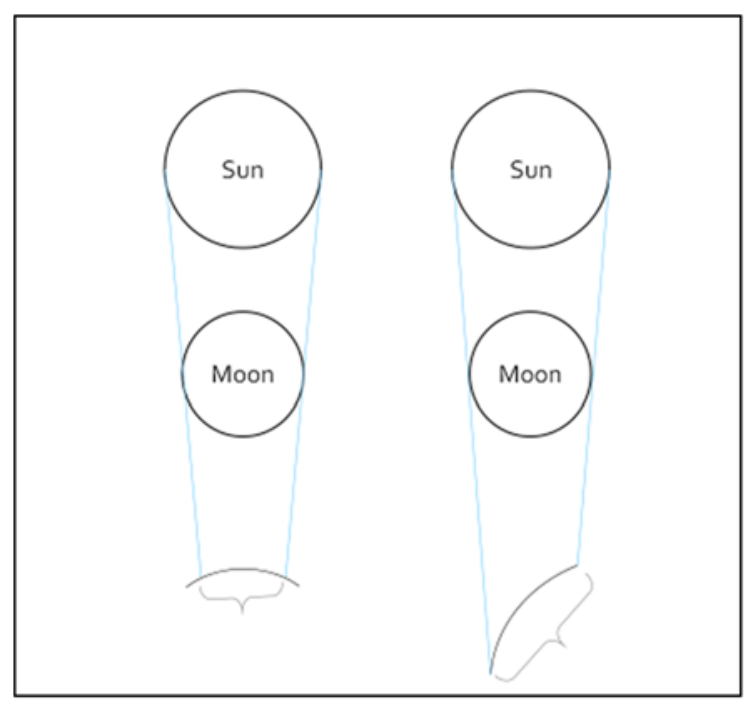

Fig. 3. The path of totality is wider towards the edges of the globe

\subsubsection{Duration of a total eclipse}

A total eclipse is divided into 3 phases demarcated by 4 events. The 3 phases in sequential order are: a Partial phase, where the Moon is partially covering the Sun; Totality where the Sun is covered by the Moon; and another Partial phase where the Moon is uncovering the Sun. The 4 events are named 1st, 2nd, 3rd, and 4th Contact. The 1st Contact is when the Moon first impinges upon the Sun's disc, signifying the start of the eclipse; 2nd Contact occurs when the Moon completely occludes the Sun, signifying the start of totality; 3rd Contact is when the Moon just uncovers the Sun, signifying the end of totality; and 4th contact is when the Moon leaves the Sun's disc, signifying the end of the eclipse. The duration from 1st to 4th contact typically lasts about 2 to 2.5 hours, and the duration from 2nd to 3rd contact can is at most about 6.5 minutes, with a theoretical minimum of 0 minutes (i.e. the Moon/Sun ratio is 1.0 so the Moon literally covers and leaves the Sun at the same time).

\subsection{Eclipse Considerations}

To observe an eclipse, the obvious factors are to have the observer at the right place (on the track/path of the eclipse) and at the right time. We shall discuss a few more considerations to be able to observe a total eclipse successfully.

\subsubsection{Accessibility of Location.}

Unfortunately, an eclipse's path does not always fall on land. In fact, statistically, the odds are that $70 \%$ of eclipse paths would be over water ${ }^{\S}$. This in and of itself, does not mean that the eclipse would not be observable, as vessels like cruise liners and smaller vessels can always be used as an observation platform, though they are less than ideal due to the motion of the waves. Even when over land though, the area may be difficult to get to, and can include areas such as the Antarctic pole as well as deep in the Amazon jungle.

$\S$ Ratio of land to sea is $3: 7$ 


\subsubsection{Safety and Security.}

Observation sites are chosen based on the level of safety and security they are able to afford. An obvious choice here would be in or near cities, and where there are no wars or other conflicts occurring. The political alignment of the country is also important to consider. Some countries are also particular about licensing and other regulatory requirements, as permits for travelling, observing, and even importation of equipment for use during the eclipse will need to be addressed.

\subsubsection{Weather.}

One of the most critical considerations in viewing an eclipse is the weather prospects, and in particular the cloud probability. Good statistical data for cloud cover for the relevant date/time of the year should be considered. Other weather considerations include the temperature, precipitation, and wind, as all these can lead to adverse viewing conditions.

\section{Eclipse Oddities}

During the phases of a total solar eclipse, there are many observational oddities due to the unique changes in lighting, as well as the optical elements because of the position of the Sun/Moon. These can be sub-divided into 2 main categories.

\subsection{Sun/Moon effects}

These are effects that can be observed purely from the juxtaposition of the Moon and the Sun.

\subsubsection{Diamond Ring and Bailey's Beads}

These 2 effects are roughly very similar though Bailey's Beads are for the more "serious" photographers and Diamond Rings are more for the lay public. They occur at 2nd and 3rd contact only, and lasts for a very short time, in the order of seconds or less. The diamond ring is primarily the effect of the glare from the Sun peeping out behind the silhouette of the Moon. Exposure settings are not as critical and is easily captured by most photographers as settings are typically overexposed. Bailey's beads on the other hand, are due to the light from the Sun peeping through the valleys at the Moon's limb, and requires very accurate exposures so that the detail of individual "beads" are not lost. Scientific data can be derived from images of Bailey's beads as they aid in determining or confirming the profile of the lunar surface at the positions of the beads.

\subsubsection{Corona}

Totality can be viewed with the naked eye without fear of any injury (unlike the rest of the partial phases, where solar filters must be used). And with the naked eye, the Sun's coronal streamers are clearly visible and seem to come alive. These streamers will appear to radiate quite far from the Sun, from 1 to 5 solar diameters. However, capturing the coronal streamers photographically is a challenge because the exposure settings vary quite dramatically depending on how close the streamers are to the Solar Disc. At its closest, the corona is very bright, and exposure settings of $1 / 8000 \mathrm{sec}, \mathrm{f} / 16$, and ISO $800 \mathrm{can}$ be used, whereas further out from the Sun, the corona is dim, and settings such as $16 \mathrm{sec}, \mathrm{f} / 4$, and ISO 100 may be 
required. A common practice is to photograph by bracketing - taking many pictures with a range of exposures. To then convey the coronal streamers and its structure after the eclipse, multiple photos will need to be processed together to extract the relevant information from the photos and reconstruct the coronal streamers that the naked eye so easily sees (because of the human eyes' high dynamic range).

\subsubsection{Chromosphere and Prominences}

Most think of the Sun as bright white in colour, so during totality, those who see a reddish colour fringing the edges of the Moon think that they are imagining it. This is in fact the chromosphere, which is a thin shell of reddish/pink colour. The colour is due to the transition Hydrogen atoms and occurs just beneath the coronal layer, and is visible only during a total eclipse. Prominences, which appear as pink "flares" emerging from the chromosphere can also be seen. Capturing the chromosphere and prominences are also challenging as a camera's exposure settings have to be low enough so that the light from the rest of the Sun does not wash out the colour and the detail of the chromosphere and prominences.

\subsubsection{Face of the Moon}

An astute observer may be able to discern that the Moon is not just a black silhouette during totality, and in fact, has some features. Light from the Sun does not directly illuminate the face of the Moon as seen from Earth. It is actually reflected off the surface of the Earth, reflected yet again from the face of the Moon, to finally reach an observer back on Earth. In order to capture this feature, cameras have to be set to very long exposures, yet not too long which may result in light from the Sun blowing out details at the edge of the Moon.

\subsection{Terrestrial and other effects}

Apart from the Sun and Moon, there are many other observational oddities, both on land as well as in the sky, that can only be seen during a total eclipse. Observers are often so entranced with the sight of totality that they fail to look away and observe the other effects to the sides and behind them.

\subsubsection{Sky colour and shape of colour}

Daytime sky illumination, which is owing to light rays from the Sun scattering off atmospheric particles, no longer occurs. But this is only true within the umbral shadow of the eclipse. A reduced proportion of light rays are still present in the penumbral shadow, and so too for the light outside of the penumbral shadow, and these rays of light can still reflect off the atmosphere back to an observer in the umbral shadow. The resultant effect is almost similar to twilight, but is also very distinct from it, with colours of the rainbow, not on the horizon, but bowl-shaped. This is because the umbral and penumbral shadows are coneshaped and when projected through the atmosphere results in a characteristic up-turned curvature.

\subsubsection{Stars and planets}

During totality, one literally experiences night in the middle of the day. And because of the darkened sky, the brighter planets and stars can often be seen. Photographers can use the aid 
of planetarium apps or programs to make sure that they are able to select the correct field of view in order to capture these specks of light during totality.

\subsubsection{Crescent sun projections}

This effect is the only one on this list that is not visible during totality, but during the partial phases. It can also be seen during annular eclipses and partial eclipses. It is still a very interesting effect and is due to the projection of the partially eclipsing Sun through pin-holes. The pin-hole can be either man-made, as in a small hole pierced through a sheet of cardboard, or naturally occurring, as in holes through foliage. The projected image can be disconcerting as the Sun appears normally bright with the naked eye, and the projected image of a crescentshaped Sun is often associated with the Moon. This effect can best be captured by having the image projected onto a flat, darkened screen that would improve the image's contrast.

\subsubsection{Shadow bands}

One of the most elusive effects are bands of shadow that race across the ground just before 2nd contact, and just after 3rd contact. These ripples of shadow are explained as being analogous to the ripples of light and shadow at the bottom of a swimming pool on a bright day. The atmosphere behaves as a fluid, as does the swimming pool's water, and refracts the light from the eclipsing Sun. The narrowness of the light from the Sun makes the refraction noticeable. This effect is best observed by laying out a flat white sheet on the ground, so that the contrast of the bands of shadow can be seen.

\section{Capture Parameters}

\subsection{Location in Sky}

Eclipses occur at varying times of the day, and therefore varying locations in the sky. The data for an eclipse can be obtained from sites like those from NASA[1]. Picking a site on the track of an eclipse will then provide valuable information including the site's latitude and longitude, the duration of totality, the date and times in UTC, and the altitude and azimuth of the various phases of the eclipse. For an eclipse lasting about 2 hours, the Earth will have rotated about 30 degrees, so a tracking mount would be helpful, but not mandatory, to counteract this movement.

With the Altitude and Azimuth of the 1st and 4th contacts, photographic and videographic equipment can be oriented to make sure that fields of view are appropriate, including watching out for obstacles or other problems. There are apps for phones available that will assist in this setup by using augmented reality to project the path of the eclipse onto the sky.

\subsection{Chronology of Events}

As the duration of a total solar eclipse is so short, understanding the sequence of events, many of which may be happening simultaneously, is critical to success. Mapping the events over the course of the eclipse is very informative as to how to capture the eclipse. Table 1 depicts when each of the effects discussed occur as the eclipse progresses. The effects marked with diamonds in their row occur at or around 2 nd and 3rd contact and are the most difficult to capture because of their brevity. The effects that occur during totality are next in line as the time to capture them are also relatively short. Effects during the partial phases can be done at leisure as these 2 phases last about an hour each. Even then, the progress of the eclipse 
Table 1. Chronology of Events

\begin{tabular}{|c|c|c|c|}
\hline \multicolumn{2}{|c|}{$1^{\text {st }}$ Contact } & $2^{\text {nd }}$ Contact & $3^{\text {rd Contact }}$ \\
\hline & Partial Phase & Totality & Partial Phase \\
\hline Duration & $\sim 1$ hour & 0.6 .5 minutes & $\sim 1$ hour \\
\hline \multicolumn{4}{|l|}{ Corona } \\
\hline Diamond Ring & \multicolumn{2}{|c|}{$\diamond$} & $\diamond$ \\
\hline Bailey's Beads & \multicolumn{2}{|c|}{$\diamond$} & $\diamond$ \\
\hline \multicolumn{4}{|l|}{ Chromosphere } \\
\hline \multicolumn{4}{|l|}{ Prominences } \\
\hline \multicolumn{4}{|c|}{ Face of Moon } \\
\hline \multicolumn{4}{|c|}{ Sky Colour, Shape of Colour } \\
\hline \multicolumn{4}{|l|}{ Stars, Planets } \\
\hline \multicolumn{4}{|c|}{ Crescent Sun Projections } \\
\hline Shadow Bands & & & \\
\hline
\end{tabular}

marches on incessantly, so if images every 5 minutes were desired, these phases can still be pretty busy for the avid eclipse capturer.

\subsection{Field of View and Speed of Capture}

The various effects span a very wide range of field of views. Sky views are the widest, and wide-angle lenses and even fish-eye lenses can be used to capture these. Prominences would probably be on the other end of the scale, as they require super-telephoto lenses.

Similarly, the duration of each effect, and the time needed to capture the effect, varies just as much. During the partial phases, solar filters that allow only a very small percentage of light through, must be used. Even then, capturing the eclipsing Sun is relatively easy as the light from the Sun is abundant, even when it is almost totally occluded. Exposure times can be very short. However, during totality, solar filters should be removed as the amount of light falls dramatically. Exposure times vary significantly depending on what is being captured. Bailey's beads are fleeting, and needs to be captured with very short exposures and a high frame rate to ensure the right moment is caught. Capturing the dim outer edges of the corona requires long exposures of up to 30 seconds (10 seconds if not using a tracking mount).

The range of fields of view, and recommended camera focal lengths, for regular $35 \mathrm{~mm}$ cameras at ISO 100, are provided in Table 2. As can be seen, some effects are best captured using video instead of photographs.

\subsection{Equipment Considerations}

Consolidating all the various effects to be captured, a range of equipment can be utilised.

\subsubsection{Tracking Mounts, Tracking Heads, Tripods}

Astronomical mounts including equatorial and Alt-Az mounts with their 1.5 or 2 inch tripods can be used effectively to track and capture the eclipse. However, these are sometimes not practical as they tend to be heavier and bulkier, and locations may be remote with limitations on luggage. Tracking heads are much more portable, but they may struggle with some of the equipment loads they need to support. A compromise is always needed for eclipses to provide the right combination. Aluminium tripods can be paired with the tracking heads, but for 
Table 2. Recommended parameters

\begin{tabular}{|l|c|c|c|c|}
\hline & Duration & Field of View & $\begin{array}{c}\text { Recommended } \\
\text { Eff Focal Length }\end{array}$ & $\begin{array}{c}\text { Recommended } \\
\text { Speeds }\end{array}$ \\
\hline $1^{\text {st }}$ to $4^{\text {th }}$ Contact & $\sim 2$ hours & Zoom & $\sim 1000 \mathrm{~mm}$ & Video \\
\hline Corona & $0-6.5 \mathrm{~min}$ & Zoom & $200-500 \mathrm{~mm}$ & $1 / 8000-30 \mathrm{sec}$ \\
\hline Diamond Ring & $<30 \mathrm{sec}$ & Zoom & $-1000 \mathrm{~mm}$ & $1 / 2000 \mathrm{sec}$ \\
\hline Bailey's Beads & $<1 \mathrm{sec}$ & Zoom & $>2000 \mathrm{~mm}$ & $1 / 8000 \mathrm{sec}$ \\
\hline Chromosphere & $0-6.5 \mathrm{~min}$ & Zoom & $\sim 1000 \mathrm{~mm}$ & $1 / 2000 \mathrm{sec}$ \\
\hline Prominences & $0-6.5 \mathrm{~min}$ & Zoom & $>2000 \mathrm{~mm}$ & $1 / 2000 \mathrm{sec}$ \\
\hline Face of Moon & $0-6.5 \mathrm{~min}$ & Zoom & $-1000 \mathrm{~mm}$ & $10-30 \mathrm{sec}$ \\
\hline $\begin{array}{l}\text { SkyColour, Shape of } \\
\text { Colour }\end{array}$ & $\sim 2$ hours & Wide & $12-35 \mathrm{~mm}$ & $1 / 60 \mathrm{sec}$ \\
\hline Stars, Planets & $0-6.5 \mathrm{~min}$ & Wide & $18-35 \mathrm{~mm}$ & $\sim 5 \mathrm{sec}$ \\
\hline $\begin{array}{l}\text { Crescent Sun } \\
\text { Projections }\end{array}$ & $0-6.5 \mathrm{~min}$ & Normal & $50-100 \mathrm{~mm}$ & $1 / 60 \mathrm{sec}$ \\
\hline Shadow Bands & $-10 \mathrm{sec}$ & Normal & $35-50 \mathrm{~mm}$ & Video \\
\hline
\end{tabular}

superior rigidity and portability, carbon fiber tripods are the way to go, at the expense of a heftier price tag.

\subsubsection{DSLR cameras, CCD cameras, Lenses, Telescopes}

The lens/sensor combination is extremely wide, with many brands and models that can be used. Even smartphone cameras can be used by many to capture the event, although they will never be able to match the size of lenses and sensor sensitivities of the larger equipment. Most DSLRs have a bracketing function, and this is very useful to capture events like Bailey's Beads and the Corona. CCDs would best be paired with a laptop (which can be a burden and an added point of failure) with software that can automate the capture process. Telescopes can be paired with CCDs or DSLRs, with afocal, prime or projection configurations all possible depending on the equipment available. Most simple refracting telescopes are usable, beginning from smaller ones with apertures of $50 \mathrm{~mm}$, with apochromatic telescopes providing better pictures due to the reduced chromatic aberrations.

When using DSLR cameras, it is best to capture photographs in raw mode, especially for the chromosphere, corona, and prominences. The dynamic ranges of these objects are very high so capturing in raw ensures that there is at least the ability to push the limits of processing to coax out the smallest of details.

\subsubsection{Cameras}

These cameras are excellent for capturing wide scenes, including effects on the ground (reaction of people, sounds, behaviour of fauna) as well as in the sky (colour of sky, changes in colours, 2nd Contact through 3rd Contact). It is best to locate these cameras at about head height (so that it captures views that appear to be from human height) and be mounted on monopods.

\subsubsection{Drones}

Drones are a relatively recent technological advancement. They provide incredible views from above and are amazingly intuitive to control. There are many legal restrictions with drones so understanding the region's regulations are important before using them. There are 
also many safety and ethical considerations for their use, including the fact that drones may obstruct, interfere or just ruin other observers' eclipse experiences. However, the ability to capture a terrestrial view from up high provides a stunning way to capture the eclipse effects on the environment, so navigating some of these obstacles are certainly worthwhile.

\subsubsection{Webcams and Smartphones cameras}

Smartphones are currently one of the more ubiquitous devices. Their photographic capability improves significantly year over year, and they even boast having 3 lenses with different fields of view in a single device. They are excellent for providing a whole variety "B-roll" footage of eclipses, and can also perform more complex tasks like time-lapse, hyper lapse, and panoramic images. The newer webcams also feature some of the newer sensors and are also getting very capable. Live streaming is an excellent, and also a relatively recent way of sharing the eclipse experience. However, an internet connection would be required, so this depends on whether the location would have this available.

\subsubsection{Action Cameras}

The small action cameras (most notably GoPro-type cameras) provide an excellent way to document eclipses. As they typically have wide angle lenses to capture more of the action, they are more suited to environmental captures. There are some newer configurations where zoom lenses can be fitted to these cameras to allow capturing Sun/Moon effects, but they are still very new and in its infancy as of the time of this writing.

\subsection{Technological Aids}

As technology progresses by leaps and bounds, the gadgets and applications that it brings are a real boon for eclipse capturing. Here are just some of the few items that are incredibly valuable to have onsite for capturing an eclipse.

\subsubsection{Internet access}

With roaming data, or even local data plans widely available in most developed or developing countries, the internet is now accessible at an observation site as long as it is not too remote. The internet is crucial for getting updated cloud cover satellite views and forecasts. If it were stable and fast enough, live streaming of the progress of eclipse becomes feasible. Realtime weather data is also important as there can be safety ramifications if storm warnings, fires, or other similar information is not available.

\subsubsection{Smartphones and Smartphone Applications}

Today's smartphones contain practically all the devices that had to be individually carried around a decade ago. GPS coordinates can now be obtained on the smartphone. Mapping software can show one's exact location, especially when inclement weather requires observers to "chase" the eclipse. Most if not all smartphones include a magnetometer, which can perform the role of a compass to find the correct azimuth. They would also have gravitational sensors which can function as an inclinometer to determine the altitude of the different contact positions in the sky.

A Bluetooth earbud is something that is undervalued during an eclipse, but is immensely useful especially when there is a lot going on. A simple recording can be played back to cue 
either a countdown to the different contacts of the eclipse, or to provide a reminder of what action is needed. For example, at 30 seconds before $2^{\text {nd }}$ contact, a reminder to "remove solar filters" can be played. An earbud (and not just audio from the smartphone's speaker) is preferred as the environment will most likely be raucous, especially when it is shared with other eclipse observers, and screams of bewilderment and excitement will drown out even the loudest of our smartphone's speakers.

Apps are significant enough that they deserve their own mention. Planetarium programs such as Sky Safari or Stellarium, are incredibly useful to simulate the eclipse beforehand, as well as to determine eclipse views when on the move (for "chasing the eclipse" scenarios). There are also eclipse apps (e.g. EclipseDroid) that provides invaluable information during eclipses. These include eclipse timings, the countdowns to contacts, simulations of eclipse views, and even augmented reality, which uses the smartphone's camera to capture the environs, and project an overlay of the eclipse's contact positions onto the actual positions in the sky. This absolutely simplifies the setting up of the fields of view of camera equipment.

Finally eclipse apps, or camera-specific apps, can go so far as to control cameras and other devices. Apps like EclipseDroid provides scripting abilities, so that a sequence of shots (e.g. to capture the corona) can be prepared in advance, and triggered when necessary. There are also intervalometer apps that can control many of the established cameras, which would be useful for time-lapse or other automated imaging capabilities.

\subsubsection{Laptops}

Laptops can be all powerful, and many have brought them to capture eclipses and control more complex equipment. However, they are power hungry, and prone to many idiosyncrasies that may make them less than reliable, especially when compared with their simpler smartphones. The challenge is always whether one can afford the hiccup during those critical few minutes, so weighing its pros and cons is going to be required.

\subsubsection{Power banks and spare batteries}

Without power, many, if not all of the devices would not be able to function, and many of their built-in batteries would also not last an entire eclipse from $1^{\text {st }}$ to $4^{\text {th }}$ contact. Thanks to advances in technology, the density of batteries, namely the lithium ion variety, is incredibly high. Power banks are relatively inexpensive and very capable at providing portable power

Table 3. Recommended Equipment

\begin{tabular}{|l|c|c|c|c|c|}
\hline & Tracking Mounts & $\begin{array}{c}\text { DSLR/CCD + } \\
\text { Telescope or Zoom } \\
\text { Lens }\end{array}$ & 360 Cameras & Drones & $\begin{array}{c}\text { Action Cams, } \\
\text { Smartphones }\end{array}$ \\
\hline $1^{\text {st }}$ to 4 $4^{\text {th }}$ Contact & $\mathrm{X}$ & $\mathrm{X}$ & $\mathrm{X}$ & $\mathrm{X}$ & $\mathrm{X}$ \\
\hline Corona & $\mathrm{X}$ & $\mathrm{X}$ & & & \\
\hline Diamond Ring & & $\mathrm{X}$ & & & \\
\hline Bailey's Beads & & $\mathrm{X}$ & & & \\
\hline Chromosphere & & $\mathrm{X}$ & & & \\
\hline Prominences & & $\mathrm{X}$ & & & \\
\hline Face of Moon & $\mathrm{X}$ & $\mathrm{X}$ & & & \\
\hline $\begin{array}{l}\text { Sky Colour, Shape of } \\
\text { Colour }\end{array}$ & & & $\mathrm{X}$ & $\mathrm{X}$ & $\mathrm{X}$ \\
\hline Stars, Planets & & & $\mathrm{X}$ & & \\
\hline $\begin{array}{l}\text { Crescent Sun } \\
\text { Projections }\end{array}$ & & & & $\mathrm{X}$ & $\mathrm{X}$ \\
\hline Shadow Bands & & & & $\mathrm{X}$ & $\mathrm{X}$ \\
\hline
\end{tabular}


in remote locations. Sealed Lead Acid batteries are also very suitable for portable power needs, but they mainly provide for devices that require $12 \mathrm{~V}$, vs the $5 \mathrm{~V}$ that power banks provide.

\section{Orchestrating the Capture}

Considering that the average duration of totality of most eclipses is about 2 to 3 minutes, and that taking a photo could take as long as 30 seconds (not even considering the time required to review and check that photographs were exposed correctly and necessary adjustments made), the importance of exposing images and capturing them with clockwork precision becomes very apparent. Also, as there are different devices that are suited for different eclipse effects, it is best to deploy several, and/or multiple devices to optimise the capture of effects throughout the eclipse.

Table 3 lists a possible assortment of the equipment that can be used for capturing the various events. As can be seen, a DSLR or CCD sensor coupled with a zoom lens or telescope provides the narrow field of view needed to capture many of the more esoteric and technically difficult aspects of the eclipse. This setup in turn would not do a good job in capturing the environmental aspects which require a wider angle of view, and simpler, more portable devices should be used instead.

Fig. 4 depicts the physical deployment of one such setup to capture the eclipse. Starting from the centre, where the observers are, are 2 narrow field devices (DSLR or CCD sensor coupled with a zoom lens or telescope), one for capturing photographs, and the other for capturing video footage. There are 2 wide field cameras for video, one in front taking in footage of the observers and the background, and another behind the observers to capture the view from the rear. A 360-camera is placed somewhere central to the observing area, and preferably with an unobstructed view all around as this footage can be used for creating VR videos.

The last element depicted in Fig. 4 is a drone in the air. The height of the drone depends on the view it is trying to capture - lower down to capture more details in the observers' reactions, or higher up to capture the changing colours in the landscape and environs. Other very important considerations for drones would require a whole separate discussion.

If there were several technically proficient operators of each device, orchestrating the capture would be a much simpler task. But more often than not, due to cost reasons, only one or two people would be operating all the devices. It is still possible for a single person to manage all the devices, provided he or she is well-versed in the various technologies. A very

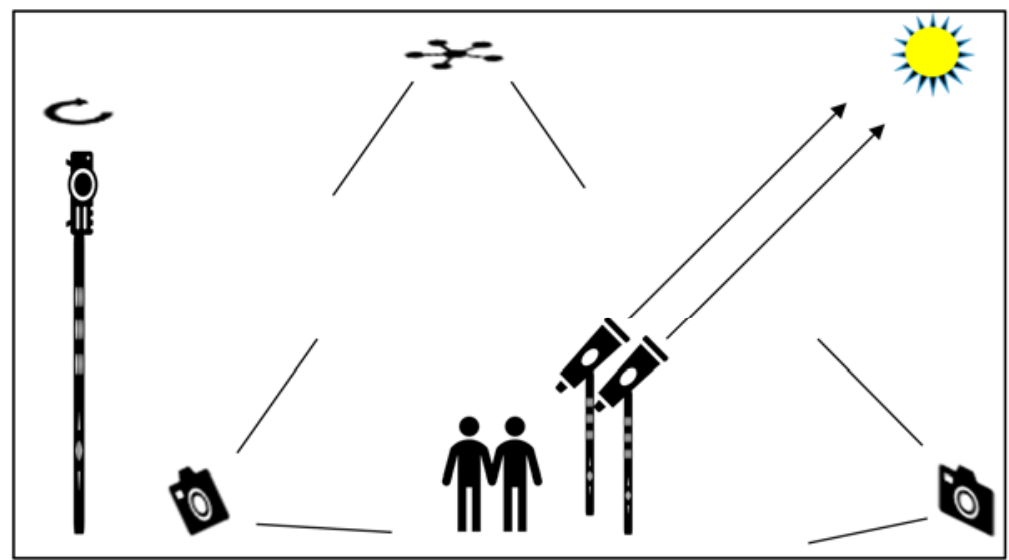

Fig. 4. Physical Deployment of Equipment 
focused and organised mind, with good diagnostic skills (as there will always be last-minute issues), are paramount in pulling this off.

Fig. 5 shows the timeline of activities that needs to be coordinated, with the majority of activities occurring about 10 minutes prior to $2^{\text {nd }}$ contact, and 5 minutes after $3^{\text {rd }}$ contact. The assumptions in this timeline are that the front and rear cameras will be continuously filming the entire eclipse from $1^{\text {st }}$ to $4^{\text {th }}$ contact, and that there will also be photographs of the progressing eclipse (which can be replaced with videos if the camera is on a tracking mount) in 5-minute intervals. Practicing the sequence of activities prior to the day, preferably with a simulation of the eclipse on a tablet or smartphone, will go a long way in making sure that all the kinks are ironed out before eclipse day. Of note in the activities listed, is the "Look at the Eclipse" reminder, since the human eye's amazing dynamic range can take in the view incredibly fast, and store that in memory, so as to recreate the experience later in post production.

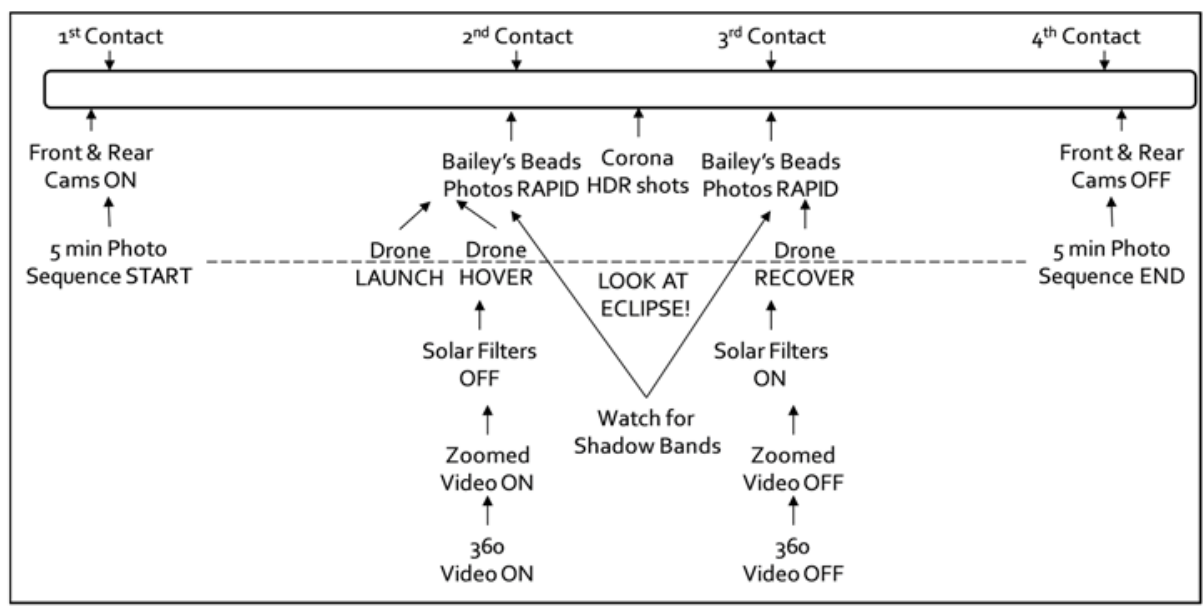

Fig. 5. Actions and Event

\section{Post processing and production}

After a successful eclipse, there is an immense satisfaction and relief as a significant amount of preparation, planning and work would have gone into capturing the fleeting event. However, the work after the eclipse, to process the photographs as well as to assemble a video to share the experience, is probably just as daunting a task for many.

\subsection{Adobe Photoshop - Photographs}

Photoshop is the gold standard for digital photography processing. Basic processing of jpg and raw files will not be discussed as there are many other sources for this information, so this should be able to create very good images (assuming of course that they were captured well) of the chromosphere, prominences, the diamond ring, and Bailey's beads. Here are some of the more unique forms of output for photos taken during an eclipse: 


\subsubsection{Eclipse GIFs}

GIFs are a form of animation, and is assembled by combining several frames of the eclipsing Sun. This is best done with a zoomed in view of the Sun, and each photo should be processed such that the Sun is always in the same size and orientation (i.e. de-rotated if taken via a nonequatorial mount). It is best to use Photoshop's guides to create 4 reference lines (top and bottom extremities and left and right edges) from an image of the Sun before $1^{\text {st }}$ contact or after $4^{\text {th }}$ contact.

All images that are to be used to create the GIF should be loaded into their own layer, and in the order in which they are to be animated - typically top to bottom representing the animation from $1^{\text {st }}$ to $4^{\text {th }}$ contact. All layers should be hidden, and the Sun on the topmost layer should be carefully centred and sized. If the Sun before $1^{\text {st }}$ contact is not available, as with locations where the eclipse has already started before it can be seen, then a reference after $4^{\text {th }}$ contact can be used.

The reference image can be toggled on and off, and its opacity modified, in order to size and align each and every image in the stack of images. This can be painstaking, but manual alignment is normally superior to the automated programs, as they aren't able to differentiate and align eclipse images which generally have very few detectable alignment features. It is important to note that it is not recommended to use the Moon as a reference for sizing and orientation. This is because the Moon, at least for total eclipses, is larger than the Sun, and it is highly likely that the Moon will be overexposed in most frames, and therefore its edges are going to be blown out and imprecise. Also, having the Sun stationary as the reference object, and having the Moon move across its face, has a more natural effect.

After all the images have been assembled, sized and aligned, the GIF can be assembled using Photoshop's "Timeline" feature. This allows all the frames to be assembled in its correct order. The timing of each frame can be manipulated to achieve various effects, and a suitable timing would be 0.5 seconds for each frame from $1^{\text {st }}$ to $2^{\text {nd }}$ contact, and $3^{\text {rd }}$ to $4^{\text {th }}$ contact, and to extend the timing during totality between $2^{\text {nd }}$ and $3^{\text {rd }}$ contact to 1 to 2 seconds or more. The GIF can also be set to infinitely loop so that it will replay itself after its sequence.

The GIF output file can then be created using "File | Save for Web", where the image type can be set to GIF. Other settings recommended would be to use the "Adaptive" colour map with 256 colours, and dither pattern of "Noise".

\subsubsection{The Solar Corona}

Recreating the solar corona that we see with the naked eye in a photograph is one of the more satisfying tasks. It is basically creating a high dynamic range image from about 8 to 10 different images with exposures ranging from $1 / 8000^{\text {th }}$ of a second to 30 seconds. The raw versions of these images should be used and processed using Adobe Camera Raw. Recommended settings are as in Table 4.

Once each image is optimised, they should be saved in 16-bit colour, and then loaded into Photoshop in individual layers and converted into smart objects. They can then be combined using the Mean function (Layer | Smart Objects | Stack Mode | Mean) and filtered (Filter | Camera Raw Filter) by adjusting for clarity, shadows and highlights. The layers can be aligned visually by nudging each layer one at a time. The Moon should be in the exact centre and set to a level that makes it all black, with any prominences showing as pink highlights.

Finally, the layers can be merged together, and then duplicated. One layer can be filtered with a radial blur filter, and using image calculations, subtract the blurred image from the original image to form an alpha channel image that can be used to sharpen the corona streamers by setting its mode to "overlay". This technique can be used more than once if desired for more sharpening. 
Table 4. Adobe Camera Raw settings

\begin{tabular}{|l|c|}
\hline Parameter & Setting \\
\hline Shadows & +83 \\
\hline Clarity & +85 \\
\hline Sharpen & $\mathbf{7 0}$ \\
\hline Radius & 25 \\
\hline Detail & 33 \\
\hline Masking & $\begin{array}{r}\text { Sharpen till noise beginsto appear, } \\
\text { then decrease belowthat. }\end{array}$ \\
\hline Luminance & 26 (or just to remove fringing) \\
\hline Detail & 50 \\
\hline Color & \\
\hline Detail & Remove Chromatic Aberration \\
\hline Lens Correction &
\end{tabular}

\subsection{Videos.}

There is a significant amount of creativity in putting together a video, and many variations can be found on the internet. The main concepts and ideas behind the video are discussed here more than the actual use of the tool. Adobe Premiere is probably one of the better tools that can be used to achieve any effect desired, though its learning curve is commensurately higher than other simpler tools.

\subsubsection{Story boarding}

It is important to decide what the video should convey to its audience, whether it is to be a personal story, a travelogue, or an educational video. Story boards are a great way to chart out the flow visually. Some common themes in these story boards include the eclipse track on the globe, the position of observation on the track, the geographical journey, the eclipse surroundings, eclipse timing and location in the sky, and of course of the actual eclipse itself.

\subsubsection{Time considerations}

As the partial phases take an hour or more in actual time, audiences would definitely be bored to watch this in real time. Having these phases compressed would create a better flow for the video, and a compression rate of 30 to 100 times would probably work well. Conversely, totality itself, and especially at the instances of $2^{\text {nd }}$ and $3^{\text {rd }}$ contacts, the actual time is very short, and goes by too fast for viewers. It is worth extending these times by either slowing them down, repeating them several times, or even replaying the same scene from different angles.

\subsubsection{Multiple source considerations}

With multiple video sources recording at the same time, some of the better video editing tools have the capability to synchronize the different sources together so that it is simple to switch 
from one view to another without losing continuity in the resulting video. In order to do this, traditional movie producers would use a clapper board. The frame where the clapper claps and the sound that is made, is used to synchronize both audio and video streams. In the case of the devices used to capture the eclipse, the best moment to "clap" is not before $1^{\text {st }}$ contact, which is what may seem to be the obvious moment. The best time to do this is actually when the last device before $2^{\text {nd }}$ contact is turned on because there are many devices with limited power (e.g. the drone), so it is best to have all devices active before the synchronization event is played. This should then be able to be used to synchronize all the streams produced.

Audio as mentioned is also an important stream, and fortunately, most recording devices include an audio track as well. Only one of the many tracks though, should be used as a master track, and this should ideally be the longest (i.e. has been recording throughout) as well as the clearest. Advanced video editing software, like Adobe Premiere, do have the capability to use each source's audio track to perform the synchronization function with. The sounds recorded during an eclipse are often unique and spectacular, as the reaction of the crowd and the surroundings during totality are often missed during the eclipse, and only realised when the recording is replayed.

The last aspect to mention is colour grading, which is the blending of the different video source's individual colour palettes. Most of the advanced video editors have this function, and to enable this, it is often best to have a white sheet of cardstock displayed or visible to all the video sources, so that colour grading can be done easily. One of the more important settings for video devices is their white balance setting. Most devices will have automatic white balance, or AWB for short, to automatically adjust the white balance point. This would actually be detrimental for eclipse capturing, as the changes in colour is actually desirable. AWB should therefore be turned off for video devices, but calibrated correctly using the reference white cardstock.

\section{Summary}

This article describes many of the amazing spectacles that can be observed during a total eclipse. Some of the tools and techniques that can be used to capture a total eclipse were discussed, and these are always being refined as technology, knowledge and experience improves with each eclipse. For anyone familiar with project management and the skills involved, they should be able to recognize that these skills are exactly what is best brought to bear to orchestrate the capture of an eclipse. That, and a good grasp of technology, to maximize its use as well as the ability to rapidly diagnose and solve any issues that emerge will ensure a successful capture. It is certainly best to experience a total solar eclipse in person, but for those unwilling or unable to travel, there is no better way to experience it than through the work of others, either in their photographs or in their videos. Hopefully, the information here would contribute in some way to the success of these astronomical hunters.

\section{References}

[1] NASAweb: Solar Eclipse Page: Solar Eclipses Past and Future, https://eclipse.gsfc.nasa.gov/solar.html (accessed 15 Nov 2019) 